\title{
Occurrence and clinical significance of endocardial late potentials and fractionations in idiopathic dilated cardiomyopathy
}

\author{
BERND-DIETER GONSKA, KLAUS-PETER BETHGE, \\ HANS-REINER FIGULLA, HEINRICH KREUZER \\ From the Department of Cardiology, University of Göttingen, Federal Republic of Germany
}

SUMMARY In order to assess the occurrence and clinical significance of abnormal electrograms in idiopathic dilated cardiomyopathy, endocardial electrode mapping during sinus rhythm and programmed ventricular stimulation were performed in 52 patients with or without clinical ventricular tachycardia. Abnormal endocardial electrograms were recorded in $77 \%$ of the patients and were diffusely distributed over the entire left ventricular endocardium. No relation could be established between the occurrence of late potentials or fractionations and clinical or induced arrhythmias. Endomyocardial biopsy samples were taken from 20 patients and showed that reduced myofibril volume fraction was related to the occurrence of abnormal endocardial electrograms. Neither induced arrhythmias nor the presence of late potentials or fractionations identified patients who died of sudden cardiac death during the mean (SD) follow up of 33 (11) months.

Thus abnormal endocardial electrograms recorded during sinus rhythm in idiopathic dilated cardiomyopathy may only be interpreted as being a sign of damage to the myocardial cells.

Left ventricular endocardial electrode mapping was developed as a means of locating the origin of ventricular tachycardia before resection. So far most of those investigated by this technique have been patients with coronary artery disease with and without a history of ventricular tachycardia or myocardial infarction, ${ }^{1-3}$ patients with a normal left ventricle, ${ }^{45}$ with conduction defects, ${ }^{6}$ and with hypertrophic cardiomyopathy. ${ }^{7}$ There are limited data on patients with idiopathic dilated cardiomyopathy. ${ }^{8}$

It has been suggested that endocardial delayed electrical activity represents an electrophysiological predisposition to ventricular slow conduction. Because slow conduction is regarded as the prerequisite for reentry, and recurrent ventricular tachy-

Requests for reprints to Dr Bernd-Dieter Gonska, Medizinische Universitätsklinik, Abteilung Kardiologie, Robert-Koch-Strasse 40, D-3400 Göttingen, Federal Republic of Germany.

Accepted for publication 28 July 1987 cardia is probably a reentrant arrhythmia, the detection of delayed electrical activity was assumed to distinguish patients with ventricular tachycardia from those without. ${ }^{9}$

Ventricular arrhythmia is common in patients with dilated cardiomyopathy. ${ }^{10-12}$ Many of them die suddenly and unexpectedly. ${ }^{1314}$ Little information is available about the relation of abnormal endocardial electrograms to clinical and induced arrhythmias in this disease. Furthermore, there is uncertainty about the prognostic importance of these findings.

This prospective study was performed in unselected patients with idiopathic dilated cardiomyopathy. We tried to answer the following questions. (a) How common are abnormal endocardial electrograms in idiopathic dilated cardiomyopathy? (b) Are these signs associated with clinical and induced ventricular arrhythmias? (c) Can their occurrence be related to histological and morphological findings? $(d)$ What is the prognostic importance of these abnormal endocardial electrograms? 


\section{Patients and methods}

\section{STUDY POPULATION}

The study population consisted of 52 patients (44 men and eight women; mean age of 49 (23-67). The diagnosis of idiopathic dilated cardiomyopathy was based on the following criteria:

(a) clinical symptoms or signs of congestive heart failure that is $\geqslant$ class II of the New York Heart Association.

(b) Echocardiographical and radiological evidence of cardiomegaly (cardiothoracic ratio $>0.5$ ).

(c) Reduced left ventricular ejection fraction.

(d) Absence of coronary artery disease confirmed by selective coronary angiography.

(e) Absence of valve lesions except for mild and moderate mitral regurgitation.

(f) Absence of specific heart muscle disease, general systemic disease, hereditary and familial disorders, systemic hypertension, or cor pulmonale.

(g) Positive histological and morphological findings in cardiac biopsy specimens.

All patients had normal concentrations of serum electrolytes. Antiarrhythmic treatment had been discontinued for at least three days before the investigations. No patient was on amiodarone at that time. All patients were informed of the purpose of the study and gave their written consent.

\section{CLINICAL AND HAEMODYNAMIC FINDINGS}

Seventeen $(33 \%)$ patients were classified as New York Heart Association functional class II, 29 (56\%) as class III, and six $(11 \%)$ as class IV. The mean (1SD) left ventricular ejection fraction at cardiac catheterisation was 38 (13)\% .

The electrocardiograms taken at rest showed atrial fibrillation in 12 patients $(23 \%)$ and sinus rhythm in $40(77 \%)$. Intraventricular conduction delay was present in 25 patients (49\%). Eighteen $(35 \%)$ had complete left bundle branch block, four $(8 \%)$ left axis deviation, and three $(6 \%)$ right bundle branch block. Electrocardiographic signs of left ventricular hypertrophy were seen in $19(37 \%)$ and of hypertrophy of both ventricles in 11 patients $(21 \%)$.

\section{CLINICAL ARRHYTHMIAS}

Fourteen $(27 \%)$ patients had a history of sustained ventricular tachycardia confirmed either by an electrocardiogram taken at rest, a 24 hour ambulatory electrocardiogram, or on the electrocardiograph monitor in the intensive care unit. In all of them complex ventricular arrhythmias were also seen during 24 hour ambulatory monitoring at the beginning of the study. Of the remaining 38 patients, 19 (37\%) had 2-472 (median 20) episodes of non-sustained ventricular tachycardia consisting of 3-20 consecutive ventricular extrasystoles at a rate of 150-210 per minute. The other 19 patients showed no ventricular tachycardia: in five of them, 1-27 episodes of ventricular bigeminy were seen during monitoring, five had multiform and five uniform single ventricular extrasystoles and only three showed no ventricular arrhythmias at all. Patients with atrial fibrillation had a lower grade of arrhythmias. Only one of them had multiform ventricular extrasystoles and ventricular bigeminy.

No relation was found between the impairment of left ventricular function and the occurrence or complexity of ventricular arrhythmias. The patients with clinical sustained ventricular tachycardia had a mean (1SD) ejection fraction of $38(13) \%$, in those with non-sustained tachycardia it was $35(13) \%$, and in patients without such arrhythmias it was 41 (11) \%

PROGRAMMED VENTRICULAR STIMULATION Programmed stimulation was performed at the right ventricular apex. The stimulation protocol included the application of one and two premature extrastimuli during sinus rhythm and at five basic driven cycle lengths $\left(S_{1} S_{1}: 600,500,428,375,330 \mathrm{~ms}\right)$. Furthermore, incremental pacing with 250 beats/min for $6 \mathrm{~s}$ and $250-300$ beats/min for $12 \mathrm{~s}$ was performed. The end point of testing was either the initiation of sustained ventricular tachycardia or refractoriness.

The induced ventricular responses were defined as follows: short ventricular response, initiation of 2-4 consecutive non-paced ventricular extrasystoles; non-sustained ventricular tachycardia, initiation of $\geqslant 5$ extrasystoles with a rate of $\geqslant 100$ beats $/ \mathrm{min}$, lasting $\leqslant 30 \mathrm{~s}$, and with no intervention required for termination; sustained ventricular tachycardia, initiation of ventricular extrasystoles with a rate of $\geqslant 100$ beats $/ \mathrm{min}$, lasting $>30$ s or requiring termination by programmed stimulation or cardioversion before then because of deterioration in haemodynamic function.

\section{ENDOCARDIAL CATHETER MAPPING}

After cardiac catheterisation and before programmed ventricular stimulation, endocardial electrode mapping of the left ventricle was performed during sinus rhythm. Figure 1 shows the mapping scheme used. In each patient endocardial electrograms were recorded from 8-12 (mean 10) different catheter positions. The recording period at each site was 15-30 s with the mapping electrode in a stable position. A fixed gain $(1 \mathrm{~mm} \simeq 40 \mathrm{mV})$ and band pass filtering of $50-500 \mathrm{~Hz}$ were used.

The abnormal endocardial electrograms were 


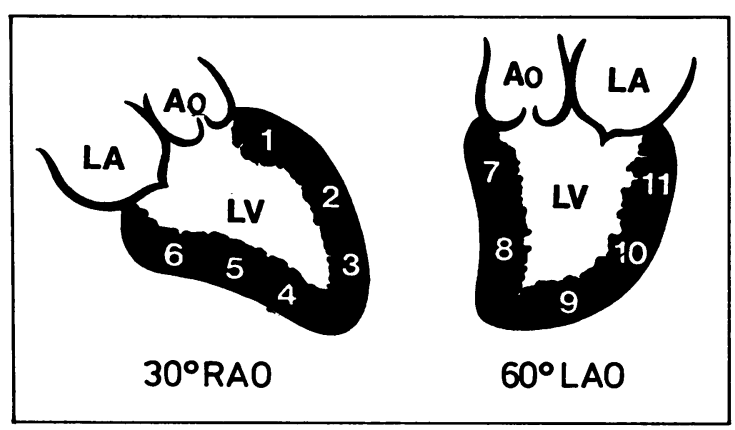

Fig 1 Mapping scheme for endocardial catheter mapping. The 11 sites represent segmental areas of the left ventricle $(L V)$ determined by $30^{\circ}$ right anterior oblique ( $R A O$ ) (sites 1-6) and $60^{\circ}$ left anterior oblique ( $L A O$ ) projection (sites 7-11). 1 = anterobasal, 2 = anteromedial, $3=$ anteroapical, $4=$ inferoapical, $5=$ inferomedial, $6=$ inferobasal, $7=$ septobasal, $8=$ septoapical, $9=$ posterolateral apical, $10=$ posterolateral medial, $11=$ posterolateral basal. LA, left atrium, $A O$ aorta.

defined as follows: late potential, well defined local deflection with an amplitude $\geqslant 100 \mu \mathrm{V} \geqslant 100 \mathrm{~ms}$ after the beginning of the QRS complex (that is after the J point); fractionation, polyphasic primarily low amplitude deflection lasting $\geqslant 50 \mathrm{~ms}$.

\section{ENDOCARDIAL BIOPSY}

Immediately after the electrophysiological studies or the day after cardiac catheterisation, biopsy samples were taken from the left ventricular wall of 20 patients by means of the King's College bioptome. The technique for estimating the volume fraction of myofibrils has been described in detail elsewhere. ${ }^{15}$

FOLLOW UP

The patients were followed up over a mean (1SD) period of 33 (11) (9-48) months. All patients with ventricular tachycardia and four out of five with ventricular bigeminy were treated with antiarrhythmic drugs at normal dosages, independent of the response to programmed ventricular stimulation (propafenone (median dose $600 \mathrm{~g}$ ), amiodarone (median dose $300 \mathrm{~g}$ ), mexiletine (median dose $600 \mathrm{~g}$ ); quinidine (median dose $1000 \mathrm{mg}$ ); flecainide (median dose $300 \mathrm{~g}$ ); sotalol (160 mg); disopyramide $(600 \mathrm{mg}))$. Treatment was started empirically and monitored by 24 hour ambulatory electrocardiography. Patients attended as outpatients. If this was not possible, their referring physicians were contacted. Sudden cardiac death was defined as cardiac death within an hour of the onset of new symptoms.

STATISTICAL ANALYSIS

Data were expressed as mean (1SD), or, when they had an asymmetrical distribution, as median and range. Statistical methods included $\chi^{2}$ analysis, the Wilcoxon $U$ test for independent samples, and the $\mathrm{H}$ test of Kruskal and Wallis. $P$ values of $<0.05$ were regarded as significant for two tailed tests.

\section{Results}

PROGRAMMED VENTRICULAR STIMULATION A repetitive ventricular response was induced in 28 $(54 \%)$ patients. In $20(39 \%)$ patients there were short responses and in eight $(15 \%)$ patients ventricular tachycardia; this was sustained in three and non-sustained in five. The rate of the tachycardia was $>200$ beats per minute in six of the eight patients. In no case was ventricular fibrillation induced directly.

The impairment of left ventricular function did not influence the result of programmed stimulation. The left ventricular ejection fraction in patients with a negative result was $37(12) \%$, in patients with $2-4$ induced ventricular extrasystoles it was $40(12) \%$, and patients with inducible ventricular tachycardia had a mean ejection fraction of $38(17) \%$.

\section{CLINICAL AND INDUCED ARRHYTHMIAS}

No clear correlation was found between the number of ventricular extrasystoles induced by programmed stimulation and the type of clinical arrhythmia; except that the induction of sustained ventricular tachycardia was only seen in patients who had sustained ventricular tachycardia clinically (table 1 ).

\section{ENDOMYOCARDIAL BIOPSY}

An endomyocardial biopsy specimen was obtained from 20 patients. None of the biopsy samples showed any evidence of myocarditis or myocardial storage disease. In 11 patients the volume fraction of myofibrils was $\leqslant 60 \%$, and in nine it was $>60 \%$. The clinical characteristics of the patients undergoing endomyocardial biopsy were no different from those of the whole study population. Left ventricular ejection fraction and the frequency of clinical or

Table 1 Results of programmed ventricular stimulation in relation to clinical arrhythmias

\begin{tabular}{lccll}
\hline \multicolumn{5}{c}{ Repetitive ventricular response } \\
\cline { 2 - 5 } & $0(\%)$ & $2-4(\%)$ & $>5(\%)$ & Total $(\%)$ \\
\hline Sustained VT & $5(36)$ & $6(43)$ & $3(21)$ & $14(100)$ \\
Non-sustained VT & $7(37)$ & $10(53)$ & $2(10)$ & $19(100)$ \\
No VT & $12(63)$ & $4(21)$ & $3(16)$ & $19(100)$ \\
Total & $24(46)$ & $20(39)$ & $8(15)$ & $52(100)$ \\
\hline
\end{tabular}

VT, ventricular tachycardia. 
Table 2 Clinical characteristics of 20 patients from whom endomyocardial biopsy samples were taken and the corresponding myofibril volume fraction

\begin{tabular}{llll}
\hline & \multicolumn{2}{l}{ Myofibril volume fraction } & \\
\cline { 2 - 3 } & $\begin{array}{l}\leqslant 60 \% \\
(n=11)\end{array}$ & $\begin{array}{l}>60 \% \\
(n=9)\end{array}$ & $\begin{array}{l}\text { Total } \\
(n=20)\end{array}$ \\
\hline $\begin{array}{l}\text { LVEF (\%) } \\
\text { Clinical arrhythmia: } \\
\text { Sustained VT } \\
\text { Non-sustained VT }\end{array}$ & $37(15)$ & $38(13)$ & $37(14)$ \\
No VT & 1 & 3 & 4 \\
RVR: & 4 & 2 & 8 \\
0 & 5 & 4 & 8 \\
$2-4$ & 5 & 3 & 8 \\
$\geqslant 5$ & - & 5 & 11 \\
\hline
\end{tabular}

LVEF, left ventricular ejection fraction; VT, ventricular tachycardia; RVR, repetitive ventricular response.

induced arrhythmias (table 2) were no different in patients with a volume fraction of myofibrils $\leqslant 60 \%$ and those with a volume fraction $>60 \%$.

\section{ENDOCARDIAL ELECTRODE MAPPING DURING} SINUS RHYTHM

Endocardial electrograms of the left ventricle were obtained from 513 electrode positions from biplanar right and left anterior oblique projections. Abnormal electrograms were recorded in $\mathbf{4 0}$ patients $(77 \%)$. Twenty eight $(54 \%)$ patients showed late potentials and $12(23 \%)$ showed late potentials and fractionations. Late potentials had a median amplitude of $340 \mu \mathrm{V}(100-500 \mu \mathrm{V})$; the median duration of fractionations was $95 \mathrm{~ms}$ (range $55-270 \mathrm{~ms}$ ).
Gonska, Bethge, Figulla, Kreuzer

Table 3 shows the frequency of late potentials and fractionations in relation to the mapping site at which they were recorded. Late potentials were found at 161 of 513 electrode positions whereas fractionations were only seen in 16 electrode positions. Both signs of delayed electrical activity were distributed over the entire endocardium of the left ventricle without being concentrated at any one site.

\section{ABNORMAL ENDOCARDIAL ELECTROGRAMS}

AND CLINICAL ARRHYTHMIAS

The occurrence of abnormal endocardial electrograms was not associated with a higher grade of ventricular arrhythmia (fig 2). Five (36\%) of 14 patients with sustained ventricular tachycardia had normal endocardial electrograms; whereas only one $(5 \%)$ patient without clinical tachycardias did not have late potentials or fractionations.

\section{ABNORMAL ENDOCARDIAL ELECTROGRAMS AND INDUCED ARRHYTHMIAS}

There were no significant differences in the occurrence of abnormal endocardial electrograms between patients with induced ventricular tachycardia, those with short repetitive responses, and those in whom the response to programmed stimulation was negative (table 4). In 20 of 40 patients with abnormal endocardial electrograms, no ventricular response could be induced; whereas, in two patients with normal endocardial electrograms, ventricular tachycardia was induced and in six short responses were initiated. All patients in whom sustained ventricular

Table 3 Occurrence of endocardial late potentials (LP) and fractionations $(F R)$ at different left ventricular mapping sites in relation to the total number of electrode positions

\begin{tabular}{|c|c|c|c|c|}
\hline Mapping site & $L P$ & $F R$ & Table (\%) & $\begin{array}{l}\text { No of catheter } \\
\text { positions }(100 \%)\end{array}$ \\
\hline $\begin{array}{l}1 \\
1-2 \\
2 \\
2-3 \\
3 \\
3-4 \\
4 \\
4-5 \\
5 \\
5-6 \\
6 \\
\text { Total }\end{array}$ & $\begin{array}{r}15 \\
7 \\
22 \\
10 \\
17 \\
8 \\
17 \\
4 \\
18 \\
8 \\
35 \\
161\end{array}$ & $\begin{array}{l}\text { Righ } \\
4 \\
-4 \\
- \\
- \\
1 \\
1 \\
\frac{3}{3} \\
16\end{array}$ & $\begin{array}{r}19(35) \\
7(54) \\
26(36) \\
10(53) \\
17(36) \\
8(47) \\
18(40) \\
5(63) \\
21(40) \\
8(30) \\
38(24) \\
177(35)\end{array}$ & $\begin{array}{r}55 \\
13 \\
73 \\
19 \\
47 \\
17 \\
45 \\
8 \\
52 \\
27 \\
157 \\
513\end{array}$ \\
\hline $\begin{array}{l}7 \\
7-8 \\
8 \\
8-9 \\
9 \\
9-10 \\
10 \\
10-11 \\
11 \\
\text { Total }\end{array}$ & $\begin{array}{r}8 \\
8 \\
20 \\
9 \\
41 \\
15 \\
33 \\
11 \\
16 \\
161\end{array}$ & $\begin{array}{l}\text { Lef } \\
1 \\
5 \\
1 \\
1 \\
4 \\
2 \\
2 \\
16\end{array}$ & $\begin{array}{r}9(15) \\
8(27) \\
25(33) \\
10(28) \\
41(46) \\
16(62) \\
37(41) \\
13(34) \\
18(28) \\
177(35)\end{array}$ & $\begin{array}{r}61 \\
30 \\
76 \\
36 \\
90 \\
26 \\
91 \\
38 \\
65 \\
513\end{array}$ \\
\hline
\end{tabular}




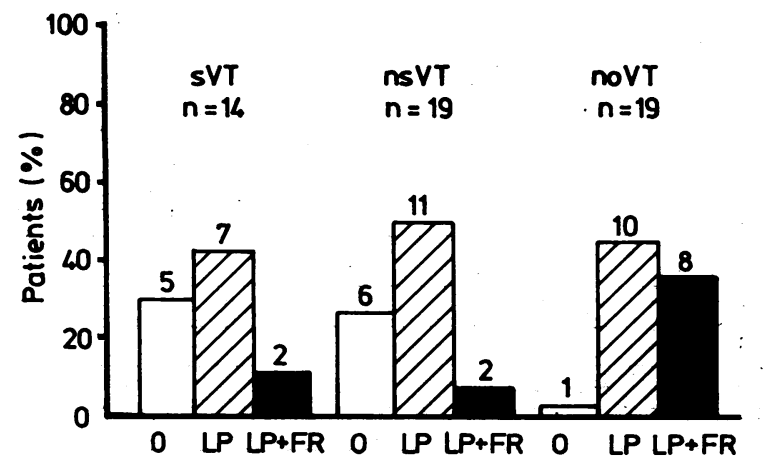

Fig 2 Endocardial late potentials ( $L P$ ) and fractionations $(F R)$ in relation to clinical arrhythmias. $s V T$, sustained ventricular tachycardia, nsVT, non-sustained ventricular tachycardia.

tachycardia was induced had abnormal endocardial electrograms.

\section{ABNORMAL ENDOCARDIAL ELECTROGRAMS}

\section{AND ENDOMYOCARDIAL BIOPSY}

Fourteen $(70 \%)$ of the 20 patients who had endomyocardial biopsy showed abnormal endocardial electrograms. The distribution of late potentials and fractionations was similar to that of the whole study population.

The volume fraction of myofibrils had a significant effect on the presence of endocardial late potentials and fractionations. Ten $(91 \%)$ of 11 patients with a myofibril volume fraction of $<60 \%$ had abnormal endocardial electrograms, whereas

Table 4 Results of programmed ventricular stimulation in relation to the presence of endocardial late potentials (LP) and fractionations ( $F R$ )

\begin{tabular}{lcccc}
\hline \multicolumn{5}{c}{ Repetitive ventricular response } \\
\cline { 2 - 5 } & 0 & $2-4$ & $\geqslant 5$ & Total $(\%)$ \\
\hline No LP or FR & $4(33)$ & $6(50)$ & $2(17)$ & $12(100)$ \\
LP & $16(57)$ & $7(25)$ & $5(18)$ & $28(100)$ \\
FR & $4(33)$ & $7(58)$ & $1(9)$ & $12(100)$ \\
Total (\%) & $24(46)$ & $20(39)$ & $8(15)$ & $52(100)$ \\
\hline
\end{tabular}

$$
\begin{gathered}
\leqslant 60 \% \\
n=11
\end{gathered} \quad \begin{gathered}
>60 \% \\
n=9
\end{gathered}
$$

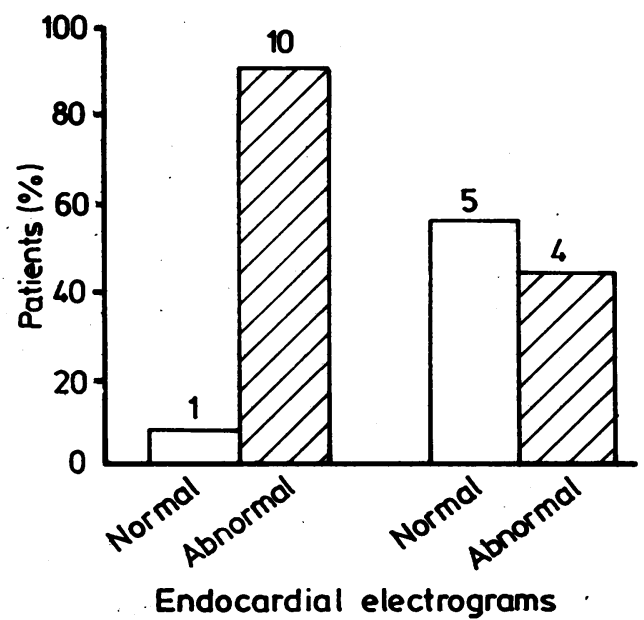

$$
\chi^{2}=4 \cdot 83>\chi_{1 ; 0.05}^{2}=3 \cdot 84
$$

Fig 3 Occurrence of abnormal endocardial electrograms in relation to the volume fraction of myofibrils in endomyocardial biopsy samples taken from 20 patients.

only four $(44 \%)$ out of nine patients with a volume fraction $>60 \%$ did $(p<0.05)$ (fig 3 ).

\section{FOLLOW UP}

During a mean follow up of 33 (11) months, nine $(17 \%)$ patients died: five of congestive heart failure, three of sudden cardiac death, and one from immunological complications after a cardiac transplant. The mean survival time (from the beginning of the study) was 15 (10) (1-25 months). Antiarrhythmic management was similar in patients who died and those who survived.

All patients who died had a significantly reduced left ventricular ejection fraction $(\leqslant 40 \%)(p<0.02)$. Three of five patients who died of congestive heart failure and all three with sudden cardiac death had

\begin{tabular}{|c|c|c|c|c|c|c|c|c|}
\hline & No & $\begin{array}{l}\operatorname{Age}(y) \\
(\text { mean }(S D))\end{array}$ & $\begin{array}{l}\operatorname{LVEF}(\%) \\
(\text { mean }(S D))\end{array}$ & $s V T(\%)$ & nsVT $(\%)$ & $R V R \geqslant 5$ & $L P$ & $L P+F R$ \\
\hline $\begin{array}{l}\text { Survivors } \\
\text { Dead: }\end{array}$ & 42 & 47 (13) & $40(13)^{\star}$ & $11(26)$ & $14(33)$ & $7(17)$ & $23(55)$ & $10(24)$ \\
\hline SCD & $\begin{array}{l}3 \\
5\end{array}$ & $\begin{array}{l}57(4) \\
49(15)\end{array}$ & $\begin{array}{ll}27 & (7) \\
31 & (9)\end{array}$ & $\begin{array}{l}1(33) \\
2(40)\end{array}$ & $\begin{array}{l}2(67) \\
1(20)\end{array}$ & - & $\begin{array}{l}1(33) \\
3(60)\end{array}$ & $\begin{array}{l}1(33) \\
1(20)\end{array}$ \\
\hline
\end{tabular}
clinical ventricular tachycardia. Programmed ven-

Table 5 Clinical characteristics of patients who died of sudden cardiac death (SCD) or of congestive heart failure (CHF) and of those who survived

${ }^{\star} \mathrm{p}<0.02$ vs those who died:

Two patients who underwent a cardiac transplant were excluded.

LVEF, left ventricular ejection fraction; sVT, sustained ventricular tachycardia; nsVT, non-sustained ventricular tachycardia;

RVR, repetitive ventricular response; LP, late potential; FR; fractionation. 
tricular stimulation induced a short response with three consecutive extrasystoles in one of the patients with sudden cardiac death and in two of the five who died of congestive heart failure. In all other patients who died, the response to programmed ventricular stimulation was negative. The incidence of abnormal endocardial electrograms was not significantly different in patients who died and those who survived (table 5).

\section{Discussion}

Endocardial catheter mapping is a newer method for evaluating abnormal endocardial electrograms. Recent studies in patients with recurrent ventricular tachycardias have focused on abnormal endocardial electrograms as the electrophysiological basis for these rhythm disturbances. So far a preponderance of patients with coronary artery disease have been investigated. Little information is available on the occurrence and clinical significance of abnormal endocardial electrograms in idiopathic dilated cardiomyopathy.

\section{CLINICAL AND INDUCED ARRHYTHMIAS}

Like others, we confirmed that ventricular arrhythmias are common in idiopathic dilated cardiomyopathy; and like Huang et $a l^{10}$ and Meinertz et $a l^{11}$ we did not find a correlation between a reduced left ventricular ejection fraction and clinical tachycardia.

There was a poor correlation between clinical and induced arrhythmias. Meinertz et al used the same stimulation protocol in $\mathbf{4 2}$ patients with dilated cardiomyopathy. ${ }^{16}$ Fifteen had clinical ventricular tachycardia, but $\geqslant 6$ ventricular extrasystoles could not be induced in any of them. None the less, they induced ventricular tachycardia in three out of 27 patients with low grade arrhythmias. Cassidy et al investigated 26 patients with idiopathic dilated cardiomyopathy in a study population of 158 patients. $^{8}$ They used a stimulation protocol of up to three extrastimuli. Twenty four of 26 patients had confirmed ventricular tachycardia or cardiac arrest. Ventricular tachycardia ( $\geqslant 3$ extrasystoles) or ventricular fibrillation was induced in $68 \%$. Thus the reproducibility of clinical ventricular tachycardia in idiopathic dilated cardiomyopathy is lower than in coronary artery disease. ${ }^{17}$

\section{ABNORMAL ENDOCARDIAL ELECTROGRAMS}

AND CLINICAL ARRHYTHMIAS

Abnormal endocardial electrograms were recorded in $77 \%$ of our study population. Fifty four per cent had late potentials and $23 \%$ had both late potentials and fractionations. A low incidence of fractionated activity was recently reported by Cassidy et $a l^{8}$-fractionated electrograms lasting $\geqslant 70 \mathrm{~ms}$ were found in only $12 \%$. Waxman and Sung ${ }^{18}$ and Josephson et al $^{19}$ reported on individual patients with idiopathic dilated cardiomyopathy and recurrent sustained ventricular tachycardia. In both of them the endocardial electrograms were abnormal.

Although we often saw abnormal endocardial electrograms and ventricular arrhythmias, there was no clear correlation between these two findings.

\section{ABNORMAL ENDOCARDIAL ELECTROGRAMS}

AND INDUCED ARRHYTHMIAS

We found that endocardial late potentials or fractionations did not influence the result obtained with programmed ventricular stimulation. Only when patients had clinical and induced sustained ventricular tachycardia was it possible to consider late potentials as the electrophysiological basis for the arrhythmia. But such an interpretation must be qualified, because in two patients without late potentials or fractionations non-sustained ventricular tachycardia was induced and in 20 patients with abnormal endocardial electrograms the response to programmed ventricular stimulation was negative. In Cassidy et al's study fractionated activity was only seen in patients with induced sustained ventricular tachycardia. $^{8}$

\section{ABNORMAL ENDOCARDIAL ELECTROGRAMS} AND ENDOMYOCARDIAL BIOPSY

The striking result was that patients with a myofibril volume fraction of $\leqslant 60 \%$ had a significantly higher occurrence of abnormal endocardial electrograms. The signs of delayed electrical activity were not concentrated at any particular site.

Studies of endomyocardial biopsy specimens have shown that the myofibril volume fraction determined in one biopsy sample is representative of the rest of the heart. ${ }^{20}$ The conclusion is that both abnormal endocardial electrograms and the reduced volume fraction of myofibrils are expressions of damage to myocardial cells.

\section{PROGNOSTIC SIGNIFICANCE OF ELECTROPHYSIOLOGICAL FINDINGS}

The main causes of death in idiopathic dilated cardiomyopathy are intractable congestive heart failure and sudden cardiac death. ${ }^{131421}$ Like other studies $^{111222}$ we found that compared with those who survived, patients who died during the mean follow up of 33 (11) months had a significantly reduced left ventricular function. There was no difference in ventricular function between patients who died of sudden cardiac death and those who died of congestive heart failure. 
Cases of sudden cardiac death could not be clearly identified by clinical arrhythmias in life. All of these patients had clinical ventricular tachycardia, but the presence of ventricular tachycardia in dilated cardiomyopathy did not identify patients with an increased risk of sudden cardiac death. Von Olshausen et al reported similar results. ${ }^{12}$ During a mean follow up of 12 (5) months, three out of 60 patients died suddenly; two of them had ventricular bigeminy. Neri et al recently confirmed this result. ${ }^{22}$

The result obtained from programmed ventricular stimulation with up to two extrastimuli was not a reliable method of predicting sudden cardiac death. Ventricular tachycardia had not been induced in any of the cases of sudden cardiac death. In the study of Meinertz et al, seven of $\mathbf{4 2}$ patients died during the follow up of 16 (7) months; two of sudden cardiac death. No patient had inducible ventricular tachycardia.

As far as we know there are no data on whether abnormal endocardial electrograms in idiopathic dilated cardiomyopathy are of prognostic importance for sudden cardiac death. In our study population, the frequency of abnormal endocardial electrograms was similar in these cases and in those who died of congestive heart failure and in those who survived.

Figulla et al recently pointed out that a reduced myofibril volume fraction was the most significant factor that influenced the haemodynamic coursedeterioration, stabilisation, or improvement. ${ }^{15}$ Because a myofibrillar volume fraction of $\leqslant 60 \%$ was more common in patients with abnormal endocardial electrograms, it might be that these findings are also related to a deterioration of haemodynamic function. This assumption is only speculative because only two of the patients who died had had an endomyocardial biopsy, and this relation was found in only one. On the other hand, two patients who died (one of sudden cardiac death and one of congestive heart failure) had normal endocardial electrograms. Further studies are required to establish whether abnormal endocardial electrograms identify patients with dilated cardiomyopathy whose haemodynamic function will worsen.

\section{Conclusion}

Abnormal electrograms recorded by means of endocardial electrode mapping during sinus rhythm are common in patients with dilated cardiomyopathy. We found there is no clear correlation between their occurrence and clinical or induced ventricular arrhythmias. Abnormal endocardial electrograms do not seem to be of prognostic value for sudden cardiac death. Therefore, it is doubtful whether 
they represent the electrophysiological basis of ventricular arrhythmias in dilated cardiomyopathy. Our results suggest that they may merely be a sign of myocardial damage.

\section{References}

1 Gallagher JJ, Kasell JH, Cox JL, Smith WM, Ideker RE, Smith WM. Techniques of intraoperative electrophysiological mapping. Am J Cardiol 1982;49:221-40.

2 Josephson ME, Horowitz LN, Spielman SR, Waxman HL, Greenspan AM. Role of catheter mapping in the preoperative evaluation of ventricular tachycardia. Am J Cardiol 1982;49:207-20.

3 Wiener I, Mindich B, Pitchon R. Determinants of ventricular tachycardia in patients with ventricular aneurysms: results of intraoperative epicardial and endocardial mapping. Circulation 1982;65:856-61.

4 Cassidy DM, Vassallo JA, Marchlinski FE, Buxton AE, Untereker WJ, Josephson ME. Endocardial mapping in humans in sinus rhythm with normal left ventricles: activation patterns and characterization of electrograms. Circulation 1984; 70:37-42.

5 Durrer D, van Lier AAW, Büller J. Epicardial and intramural excitation in chronic myocardial infarction. Am Heart $J$ 1964;68:765-76.

6 Vassallo JA, Cassidy DM, Marchlinski FE, et al. Endocardial activation of left bundle branch block. Circulation 1984;69:914-23.

7 Kuck KH, Kunze KP, Schlüter $M$, Geiger $M$, Bleifeld W. Endocardial mapping during sinus rhythm in patients with hypertrophic cardiomyopathy [Abstract]. Eur Heart J 1985;6(suppl 1):18.

8 Cassidy DM, Vassallo JA, Miller JM, et al. Endocardial catheter mapping in patients in sinus rhythm: relationship to underlying heart disease and ventricular arrhythmias. Circulation 1986;73:645-52.

9 Wit AL, Allessie MA, Bonke FJM, Lammers W, Smeets J, Fenoglio JJ Jr. Electrophysiologic mapping to determine the mechanism of experimental ventricular tachycardia initiated by premature impulses. Experimental approach and initial results demonstrating reentrant excitation. Am J Cardiol 1982;49: 166-85.

10 Huang SK, Messer JV, Denes P. Significance of ventricular tachycardia in idiopathic dilated cardiomyopathy: observations in 35 patients. $\mathrm{Am} \mathrm{J}$ Cardiol 1983;51:507-12.

11 Meinertz T, Hofmann T, Kasper W, et al. Significance of ventricular arrhythmias in idiopathic dilated cardiomyopathy. Am J Cardiol 1984;53:902-7.

12 von Olshausen K, Schäfer A, Mehmel HC, Schwarz F, Senges J, Kübler W. Ventricular arrhythmias in idiopathic dilated cardiomyopathy. $\mathrm{Br}$ Heart $\mathrm{J}$ 1984;51:195-201.

13 Goodwin JF. Prospects and predictions for the cardiomyopathies. Circulation 1974;50:210-9.

14 Hatle L, Orjavik O, Storstein O. Chronic myocardial disease. I. Clinical picture related to long-term prognosis. Acta Med Scand 1976;199:399-405. 
15 Figulla HR, Rahlf G, Nieger M, Luig H, Kreuzer H. Spontaneous hemodynamic improvement or stabilization and associated biopsy findings in patients with congestive cardiomyopathy. Circulation 1985; 71:1095-104.

16 Meinertz T, Treese N, Kasper W, et al. Determinants of prognosis in idiopathic dilated cardiomyopathy as determined by programmed electrical stimulation. Am J Cardiol 1985;56:337-41.

17 Gonska BD, Bethge KP, Kreuzer H. Programmed ventricular stimulation in coronary artery disease and dilated cardiomyopathy: influence of the underlying heart disease on the results of electrophysiologic testing. Clin Cardiol 1987;10:294-304.

18 Waxman HL, Sung RJ. Significance of fragmented ventricular electrograms observed using intracardiac recording techniques in man. Circulation 1980;62:
1349-56.

19 Josephson ME, Horowitz LN, Farshidi A, Spear JF, Kastor JA, Moore EN. Recurrent sustained ventricular tachycardia. 2. Endocardial mapping. Circulation 1978;57:440-7.

20 Figulla HR, Rahlf G, Bardosi A, et al. Histomorphometrische Untersuchungen bei Patienten mit dilatativer Kardiomyopathie [Abstract]. Z Kardiol 1986;75(suppl 1):15.

21 Segal JP, Stapleton JF, McClellan JR, Waller BF, Harvey WP. Idiopathic cardiomyopathy: clinical features, prognosis and therapy. In: Harvey WP, ed. Current problems in cardiology. Chicago: Year Book Medical Publishers, 1978:1-49.

22 Neri R, Mestroni L, Salvi A, Camerini F. Arrhythmias in dilated cardiomyopathy. Postgrad Med J 1986;62:593-7. 